\title{
Tunneling Conductance in Normal-Insulator-Superconductor junctions of Silicene
}

\author{
Surajit Sarkar ${ }^{1}$, Arijit Saha ${ }^{2,3}$ and Suhas Gangadharaiah ${ }^{1}$ \\ 1 Department of Physics, Indian Institute of Science Education and Research, Bhopal, India \\ ${ }^{2}$ Institute of Physics, Sachivalaya Marg, Bhubaneswar, Orissa, 751005, India \\ ${ }^{3}$ Homi Bhabha National Institute, Training School Complex, Anushakti Nagar, Mumbai 400085, India
}

(Dated: September 5, 2016)

\begin{abstract}
We theoretically investigate the transport properties of a normal-insulator-superconductor (NIS) junction of silicene in the thin barrier limit. Similar to graphene the tunneling conductance in such NIS structure exhibits an oscillatory behavior as a function of the strength of the barrier in the insulating region. However, unlike in graphene, the tunneling conductance in silicene can be controlled by an external electric field owing to its buckled structure. We also demonstrate the change in behavior of the tunneling conductance across the NIS junction as we change the chemical potential in the normal silicene region. In addition, at high doping levels in the normal region, the period of oscillation of the tunneling conductance as a function of the barrier strength changes from $\pi / 2$ to $\pi$ with the variation of doping in the superconducting region of silicene.

PACS numbers: 73.23.-b, 73.63.-b, 74.45.+c, 72.80.Vp
\end{abstract}

\section{INTRODUCTION}

One of the most active research field in condensed matter physics since the last decade has been the study of Dirac fermions in graphene ${ }^{1}$ and topological insula$\operatorname{tor}^{2,3}$. The low energy spectrum of these materials satisfies massless Dirac equation. The relativistic band structure of the Dirac fermions has lead to tremendous interest in graphene in terms of possible application as well as from the point of view of fundamental physics.

Very recently, a silicon analogue of graphene, silicene has been attracting a lot of attention both theoretically and experimentally ${ }^{4-8}$, due to the possibility of new applications, given its compatibility with silicon based electronics. Unlike graphene, silicene does not have a planar structure; instead the buckled structure of silicene manifests itself as a spin-orbit coupling resulting in a band gap at the Dirac point ${ }^{5}$. More interestingly, it has been reported earlier that such band gap is tunable by an external electric field applied perpendicular to the silicene sheet $^{9,10}$. This opens up the possibility of realizing silicene based electronics and very recently a silicene based transistor has been experimentally realized ${ }^{11}$.

In recent times, it has been realized that topologically non-trivial phases arise in silicene, tuned by the external electric field only ${ }^{10,12,13}$. Graphene and silicene have similar band structures and the low energy spectrum of both are described by the relativistic Dirac equation i.e., both have the Dirac cone band structure around the two valleys represented by the momenta $\mathbf{K}$ and $\mathbf{K}^{\prime}$. However, the important difference between graphene and silicene is that the spin-orbit coupling (SOC) in silicene is much stronger than in graphene $e^{5,10,14}$ which causes the Dirac fermions in silicene to become massive. Furthermore, due to the buckled structure in silicene, the two sub-lattices respond differently to an externally applied electric field resulting in electrically tunable Dirac mass term ${ }^{10}$. Such tunability allows for the mass gap to be closed at some critical value of the electric field and then reopened. Hence, the phases on the two sides of the critical electric field where the gap is closed are different, with one of them being topologically trivial and the other being topologically non-trivial ${ }^{10,12,13}$. As a result, silicene under the right circumstances can be a quantum spin hall insulator with topologically protected edge states ${ }^{12,15,16}$.

The advent of superconductivity in graphene and certain topological insulators via the proximity effect has led to an upsurge of interest in this area ${ }^{2,17}$. Very recently, superconducting proximity effect in silicene has been reported in Ref. 18 where the authors have investigated the behavior of Andreev reflection (AR) and crossed Andreev reflection (CAR) in a normal-superconductor (NS) and normal-superconductor-normal (NSN) junctions of silicene respectively.

In this article, we study the behavior of tunneling conductance (TC) in a normal-insulator-superconductor (NIS) junction of silicene where superconductivity in silicene is induced via the proximity effect. We model our NIS setup within the scattering matrix formalism ${ }^{19,20}$ and obtain the external electric field controllable TC for thin barrier limit. Similar set up in graphene have been studied earlier in Refs. 21 and 22. However, TC based on silicene NIS structure has not yet been considered in the literature.

The remainder of this paper is organized as follows. In Sec. II, we present our model for the silicene NIS structure and describe the scattering matrix formalism to compute the tunneling conductance. In Sec. III, we present our results for the TC in the NIS set-up for the thin barrier case. Finally in Sec. IV, we summarize our results followed by the conclusions.

\section{MODEL AND METHOD}

In this section we will set up the equations to study the transport properties of an NIS junction in a silicene sheet placed along the $x y$-plane (see Fig. 1). The $x \leq-d$ region is the normal region $(\mathrm{N})$, the insulating region (I) has a 


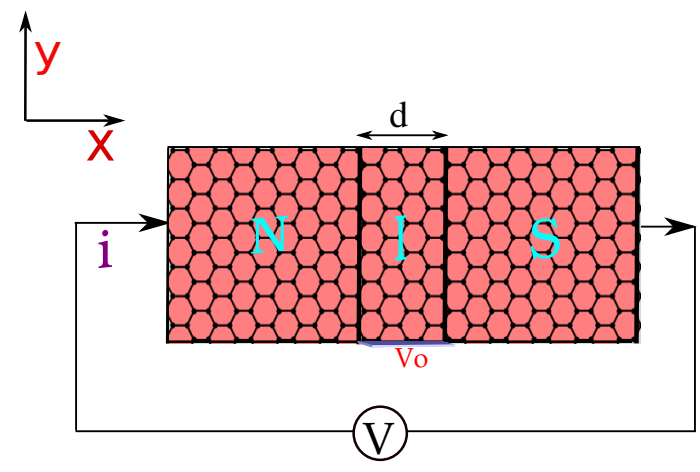

FIG. 1. (Color online) Schematic of the silicene NIS junction on an $x y$-plane. $N$ corresponds to the normal region, a barrier of height $V_{0}$ is applied in the $I$ region (with width $d$ ) to make it insulating. Superconductivity is induced in the $S$ region via the proximity effect.

width $d$ and occupies the $-d \leq x \leq 0$ region, while the superconducting region (S) occupies $x \geq 0$ region. The insulating region has a gate tunable barrier potential of strength $V_{0}$, while the superconductivity in $x \geq 0$ region is assumed to have been induced via the proximity effect where the external superconductor is taken to be of the s-wave type.

The Silicene NIS junction is described by the Dirac Bogoliubov-de Gennes (DBdG) equation of the form

$$
\left(\begin{array}{cc}
\hat{H}_{\eta} & \Delta \hat{I} \\
\Delta^{\dagger} \hat{I} & -\hat{H}_{\eta}
\end{array}\right) \Psi=E \Psi
$$

where $E$ is the excitation energy, $\Delta$ is the proximity induced superconducting energy gap. The Hamiltonian $\hat{H}_{\eta}$ describes the low energy physics near each of the $\mathbf{K}, \mathbf{K}^{\prime}$ Dirac points and has the form ${ }^{18}$,

$$
H_{\eta}=\hbar v_{F}\left(\eta k_{x} \hat{\tau}_{x}-k_{y} \hat{\tau}_{y}\right)+\left(l E_{Z}-\eta \hat{\sigma}_{z} \lambda_{S O}\right) \hat{\tau}_{z}-\mu_{i} \hat{1},
$$

where $\eta=+(-)$ corresponds to $\mathbf{K}\left(\mathbf{K}^{\prime}\right)$ valleys, $v_{F}$ is the fermi velocity (in the following we will set $\hbar v_{F}=1$ ), $\mu_{i}(i$ represents any of the $\mathrm{N} / \mathrm{I} / \mathrm{S}$ regions $)$ is the chemical potential and $\lambda_{S O}$ is the spin orbit term. The Pauli matrices $\hat{\sigma}$ and $\hat{\tau}$ act on the spin and sub-lattice space, respectively. The potential energy term $l E_{Z}$ owes its origin to the buckled structure of the Silicene wherein the $A$ and $B$ sites occupy slightly different planes (separated by a distance of length $l$ ) and therefore acquire a potential difference when an external electric field $E_{Z}$ is applied perpendicular to the plane. It turns out that at the critical electric field $E_{Z}^{c}=\lambda_{S O} / l$ each of the valleys become gapless with the gapless bands of one of the valley being up-spin polarized and the other down-spin polarised ${ }^{10,23}$. Away from the critical field, the bands (corresponding to
$\left.H_{\eta}\right)$ at each of the $\mathbf{K}$ and $\mathbf{K}^{\prime}$ points are split into two conduction and two valence bands with the band gap being $\left|l E_{Z}-\eta \sigma \lambda_{S O}\right|$, where $\sigma= \pm 1$ is a spin index.

Assuming translational invariance along the $y$ direction we solve Eq. (1) to find the wave functions in all the three different regions. The wave functions for the electrons and holes moving along the $\pm x$ direction in the $\mathrm{N}$ region are

$$
\begin{aligned}
& \psi_{N}^{e \pm}=\sqrt{\frac{1}{2 \tau_{1}^{e}\left(E+\mu_{N}\right)}}\left[\begin{array}{c}
k_{1}^{e} e^{ \pm i \eta \alpha_{e}} \\
\pm \eta \tau_{1}^{e} \\
0 \\
0
\end{array}\right] e^{i\left( \pm k_{1 x}^{e} x+k_{1 y}^{e} y\right)} \\
& \psi_{N}^{h \pm}=\sqrt{\frac{1}{2 \tau_{1}^{h}\left(E-\mu_{N}\right)}}\left[\begin{array}{c}
0 \\
0 \\
k_{1}^{h} e^{ \pm i \eta \alpha_{h}} \\
\mp \eta \tau_{1}^{h}
\end{array}\right] e^{i\left( \pm k_{1 x}^{h} x+k_{1 y}^{h} y\right)},(3)
\end{aligned}
$$

where $\tau_{1}^{e(h)}=E \pm \mu_{N} \mp\left(l E_{Z}-\eta \sigma \lambda_{S O}\right)$ and $E$ is the energy of the particle wrt. to the Fermi level $\mu_{N}$. We note that due to the spin being a good quantum number (and also because of time reversal symmetry) we can restrict our discussion by considering spin of only one type.

The conservation of momentum along the $y$-direction, $k_{1 y}^{e}=k_{1 y}^{h}$, leads to the angle of incidence $\alpha_{e}$ and the Andreev reflection angle $\alpha_{h}$ being related via, $k_{1}^{h} \sin \left(\alpha_{h}\right)=$ $k_{1}^{e} \sin \left(\alpha_{e}\right)$ where

$$
k_{1}^{e(h)}=\sqrt{\left(E \pm \mu_{N}\right)^{2}-\left(l E_{Z}-\eta \sigma \lambda_{S O}\right)^{2}} .
$$

In the insulating region the wave functions are

$$
\begin{aligned}
& \psi_{I}^{e \pm}=\sqrt{\frac{1}{2 \tau_{2}^{e}\left(E+\mu_{I}\right)}}\left[\begin{array}{c}
k_{2}^{e} e^{ \pm i \eta \beta_{e}} \\
\pm \eta \tau_{2}^{e} \\
0 \\
0
\end{array}\right] e^{i\left( \pm k_{2 x}^{e} x+k_{2 y}^{e} y\right)} \\
& \psi_{I}^{h \pm}=\sqrt{\frac{1}{2 \tau_{2}^{h}\left(E-\mu_{I}\right)}}\left[\begin{array}{c}
0 \\
0 \\
k_{2}^{h} e^{ \pm i \eta \beta_{h}} \\
\mp \eta \tau_{2}^{h}
\end{array}\right] e^{i\left( \pm k_{2 x}^{h} x+k_{2 y}^{h} y\right)},
\end{aligned}
$$

where $\tau_{2}^{e(h)}=E \pm \mu_{I} \mp\left(l E_{Z}-\eta \sigma \lambda_{S O}\right)$ and

$$
k_{2}^{e(h)}=\sqrt{\left(E \pm \mu_{I}\right)^{2}-\left(l E_{Z}-\eta \sigma \lambda_{S O}\right)^{2}},
$$

where $\mu_{I}=\mu_{N}-V_{0}$ and $V_{0}$ is the electrostatic potential that controls the barrier height.

Finally, in the superconducting region the wave functions of $\mathrm{DBdG}$ quasiparticles are given by,

$$
\begin{aligned}
& \psi_{S}^{e}=\frac{1}{\sqrt{2}}\left[\begin{array}{c}
u_{1} \\
\eta u_{1} e^{i \eta \theta_{e}} \\
u_{2} \\
\eta u_{2} e^{i \eta \theta_{e}}
\end{array}\right] e^{\left(i \mu_{S}-\kappa\right) x+i q_{y}^{e} y} \\
& \psi_{S}^{h}=\frac{1}{\sqrt{2}}\left[\begin{array}{c}
u_{2} \\
-\eta u_{2} e^{-i \eta \theta_{h}} \\
u_{1} \\
-\eta u_{1} e^{-i \eta \theta_{h}}
\end{array}\right] e^{\left(-i \mu_{S}-\kappa\right) x+i q_{y}^{h} y},
\end{aligned}
$$


where

$$
u_{1 / 2}=\sqrt{\frac{1}{2} \pm \frac{\sqrt{E^{2}-\Delta^{2}}}{2 E}} \text { and } \kappa=\sqrt{\Delta^{2}-E^{2}} .
$$

As before, momentum conservation along the $y$-direction relates the transmission angles for electron-like and holelike quasi-particles via the following equation

$$
q^{i} \sin \theta_{i}=k_{1}^{e} \sin \alpha_{e},
$$

for $i=e, h$. The quasiparticle momentums are given by

$$
q^{e(h)}=\mu_{S} \pm \sqrt{E^{2}-\Delta^{2}}
$$

where $\mu_{S}=\mu_{N}+U_{0}$ and $U_{0}$ is the gate potential applied in the superconducting region to tune the Fermi surface mismatch.

Let us consider an electron with energy $E$ incident on the interface of a conventional NIS junction of a silicene sheet. Part of the wave function gets transmitted and the rest undergoes both normal and Andreev reflection from the interface. Taking into consideration all these processes the wave functions in the different regions of junction can be written $\operatorname{as}^{20}$ :

$$
\begin{aligned}
\Psi_{N} & =\psi_{N}^{e+}+r \psi_{N}^{e-}+r_{A} \psi_{N}^{h-} \\
\Psi_{I} & =p \psi_{I}^{e+}+q \psi_{I}^{e-}+m \psi_{I}^{h+}+n \psi_{I}^{h-} \\
\Psi_{S} & =t_{e} \psi_{S}^{e}+t_{h} \psi_{S}^{h},
\end{aligned}
$$

where $r$ and $r_{A}$ are the amplitudes of normal reflection and Andreev reflection in the $N$ region, respectively. The transmission amplitudes $t_{e}$ and $t_{h}$ correspond to electron like and hole like quasiparticles in the $S$ region, respectively. From the continuity of the wave functions at the two interfaces we have

$$
\left.\Psi_{N}\right|_{x=-d}=\left.\left.\Psi_{I}\right|_{x=-d} \quad \Psi_{I}\right|_{x=0}=\left.\Psi_{S}\right|_{x=0}
$$

which yields a set of eight linearly independent equations. Solving them we obtain $r$ and $r_{A}$, these amplitudes fully determine the tunnelling conductance of the NIS junction within the Blonder-Tinkham-Klapwijk formalism (BTK) 20

$$
\frac{G(e V)}{G_{0}(e V)}=\int_{0}^{\pi / 2}\left[1-|r|^{2}+\left|r_{A}\right|^{2} \frac{\cos \left(\alpha_{h}\right)}{\cos \left(\alpha_{e}\right)}\right] \cos \left(\alpha_{e}\right) d \alpha_{e}
$$

where $G_{0}$ is the ballistic conductance of silicene.

\section{THIN BARRIER}

In this section we will solve the scattering problem in the limiting case of thin barrier. We will consider the limit where the barrier height $V_{0} \rightarrow \infty$ while the width $d \rightarrow 0$ such that the product $V_{0} d \rightarrow \chi$ is finite and nonzero $^{21}$. We will consider only those scenarios wherein the mean-field criterion for superconductivity, i.e., $\mu_{S}=$
$\mu_{N}+U_{0} \gg \Delta$, is satisfied. This can be achieved by controlling either the doping level $\mu_{N}$ or the gate voltage $U_{0}$ in the superconducting region. Solving the boundary condition (12) for $r$ and $r_{A}$ we obtain,

$$
r=\frac{N_{R}}{D}, \quad r_{A}=\frac{N_{A R}}{D}
$$

where

$$
\begin{aligned}
D=- & e^{4 i \chi}\left(e^{i \alpha_{e}}-\gamma\right)\left(e^{i \alpha_{h}}-\delta\right)+e^{2 i \beta}\left(e^{i \alpha_{e}}+\gamma\right)\left(e^{i \alpha_{h}}+\delta\right), \\
N_{R}= & e^{i \alpha_{e}}\left[e^{4 i \chi}\left(1+\gamma e^{i \alpha_{e}}\right)\left(e^{i \alpha_{h}}-\delta\right)+e^{2 i \beta}\left(\gamma e^{i \alpha_{e}}-1\right)\right. \\
& \left.\times\left(e^{i \alpha_{h}}+\delta\right)\right],
\end{aligned}
$$

and

$$
N_{A R}=2 \tilde{A} e^{i\left(\alpha_{h}+2 \chi\right)}\left(1+e^{2 i \alpha_{e}}\right) \gamma e^{i \beta} .
$$

The remaining parameters are defined as follows,

$$
\begin{gathered}
\gamma=\sqrt{\frac{\left(\epsilon+\mu_{N}+\lambda\right)}{\left(\epsilon+\mu_{N}-\lambda\right)}} ; \quad \delta=\sqrt{\left.\frac{\left(\epsilon-\mu_{N}-\lambda\right)}{\left(\epsilon-\mu_{N}+\lambda\right.}\right)} \\
\tilde{A}=\sqrt{\frac{\left(\epsilon+\mu_{N}-\lambda\right)\left(\epsilon-\mu_{N}\right)}{\left(\epsilon-\mu_{N}+\lambda\right)\left(\epsilon+\mu_{N}\right)}}
\end{gathered}
$$

where $e^{i \beta}=u_{1} / u_{2}, \epsilon=e V / \Delta$ and $\lambda \Delta=\left(l E_{Z}-\eta \sigma \lambda_{S O}\right)$. Unless otherwise stated we consider a scenario wherein the product $\eta \sigma=1$, and the upper conduction sub-band remains unfilled.
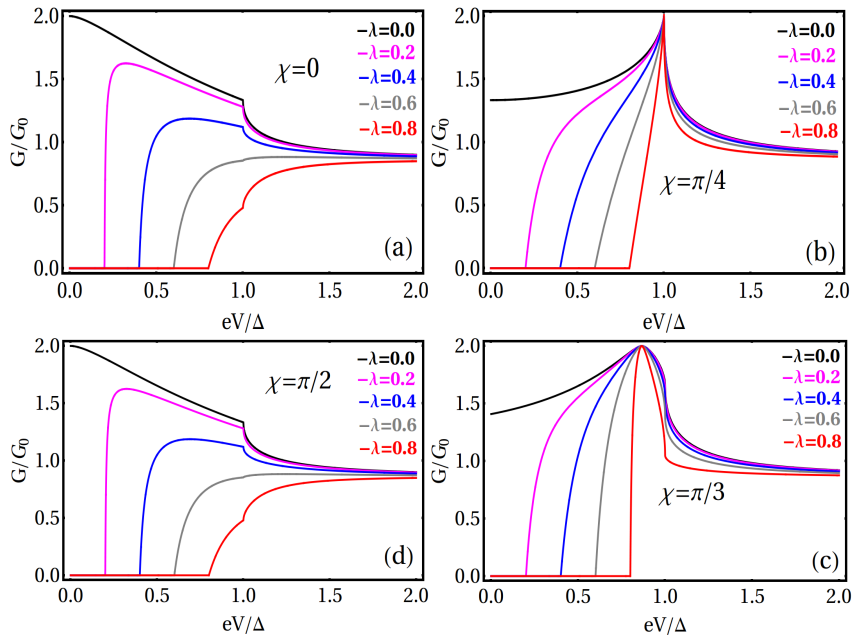

FIG. 2. (Color online) Plot of tunneling conductance of a NIS silicene junction as a function of bias voltage for different barrier strength $\chi$ and gap $\lambda$ in the undoped regime $\left(\mu_{N}=0\right)$ and for $U_{0} \gg \Delta$.

\section{A. Undoped regime}

In this subsection we focus on the undoped regime $\left(\mu_{N}=0\right)$ in the normal side of the silicene sheet. The 

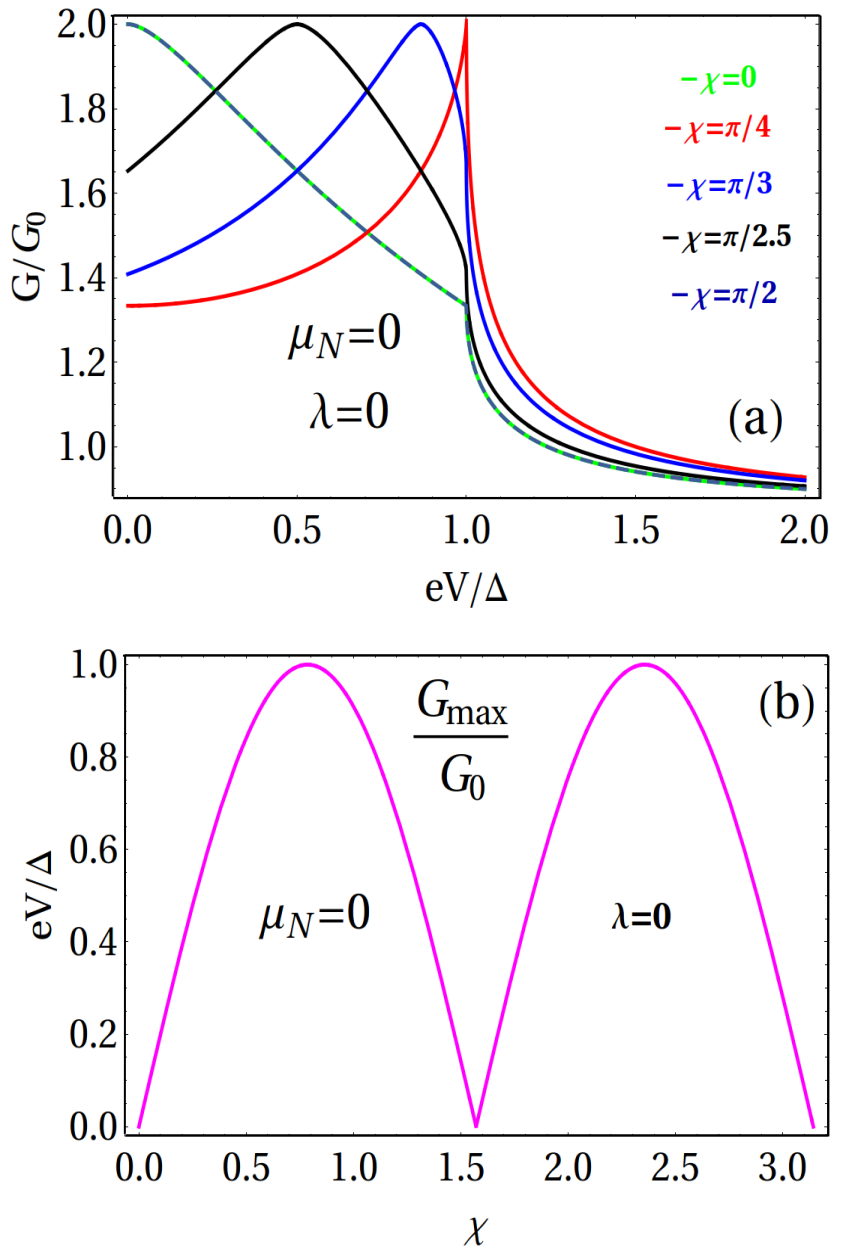

FIG. 3. (Color online) (a)Tunneling conductance as a function of bias voltage for different barrier strength at $\lambda=0$ and $U_{0} \gg \Delta$. (b) The position of the maxima of $G / G_{0}$ in the $\mathrm{eV} / \Delta$ vs $\chi$ plot.

tunnel conductance value $G / G_{0}$ varies from 0 to a peak value of 2 with the Andreev reflection contributions being purely of the specular kind. The plot of $G / G_{0}$ as a function of $e V / \Delta$ for a fixed barrier strength $\chi$ and different $\lambda$ 's are shown in Fig. 2. For the transparent barrier regime (i.e., $\chi=0$ ) we obtain plots, Fig. 2a, identical to those in Ref. (18). A common theme for $G / G_{0}$ vs $\mathrm{eV} / \Delta$ for all the different barrier strengths [see plots Figs. 2(a)-(d)] is that the non-zero contribution to conductance arises when the incident electron has energy greater than the band gap.

Interestingly, this feature can be exploited by the electric field applied perpendicular to the plane which can then be used as a switch to turn on or off the conductance. Tuning the electric field so that $\lambda=0$ brings the system to the Dirac limit and assures non-zero conductance at zero bias and for arbitrary strengths of the barrier. Another similarity between the different plots is the significant change in the slope at $e V / \Delta=1$, this can be attributed to the sudden suppression of Andreev re-

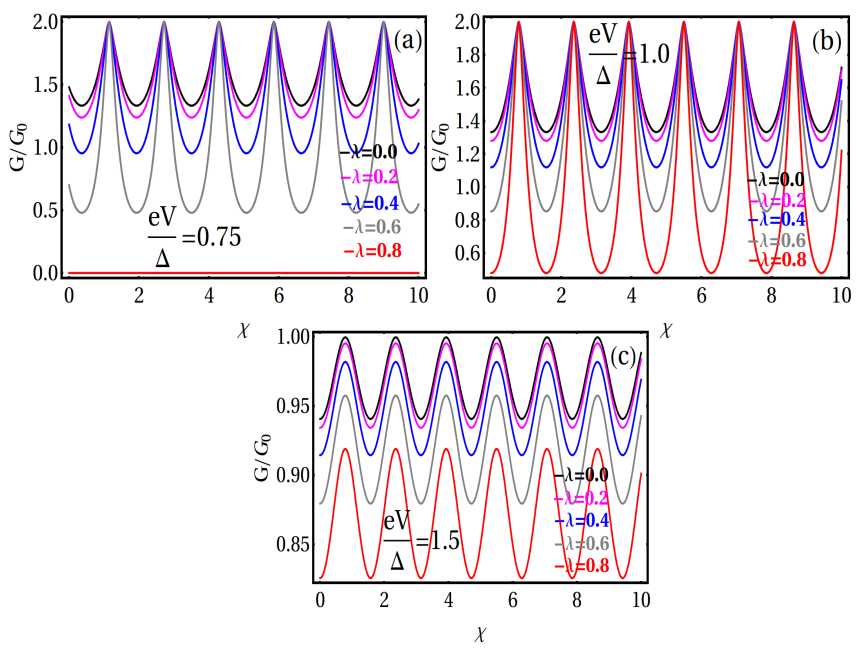

FIG. 4. (Color online) Tunneling conductance in the limit of $U_{0} \gg \Delta$ as a function of barrier strength $\chi$ for undoped regime $\left(\mu_{N}=0\right)$, (a) in the sub-gap regime, (b) $\mathrm{eV}=\Delta$ and, (c) $e V>\Delta$.

flection for electron energies beyond the sub-gap regime.

We see from the plots Figs. 2(a)-(d) that the tunnelling conductance profile and the peak positions depend significantly on the barrier strength. Nevertheless, the profile remains unchanged for barrier strengths which differ by $\pi / 2$, i.e., for $\chi \rightarrow \chi+\pi / 2$. This is understood by considering the simpler case of $\lambda=0$ for which the reflection amplitude at an arbitrary incident angle $\alpha_{e}$ has the expression

$$
r=\frac{\left(e^{2 i \alpha_{e}}-1\right) \cos (\beta-2 \chi)}{2\left[\cos (\beta-2 \chi)+i \cos \left(\alpha_{e}\right) \sin (\beta-2 \chi)\right]} .
$$

The above expression remains invariant for every $\chi$ that differs by integer multiple of $\pi / 2$. We also note that for $\lambda=0$ the peak value of TC is $G / G_{0}=2$ [see Fig. 3(a)]which is achieved when the reflection coefficient $r$ vanishes, or in other words $\left|r_{A}\right|=1$ (follows from the unitarity criterion). This is realised for all incident angles when the transmission resonance criterion is satisfied, i.e., $\cos (\beta-2 \chi)=0$. In Fig. 3(b) we plot $e V / \Delta$ vs $\chi$ for which transmission resonance condition is satisfied.

The oscillatory behavior of $G / G_{0}$ as a function of $\chi$ persists even for non-zero $\lambda$ 's. We plot this behavior in Figs. 4(a)-(c), where the plots are for a fixed $\mathrm{eV} / \Delta$ and different $\lambda$ 's. As is expected, the peak value of $G / G_{0}=2$ is achieved for incoming electrons whose energy is below the sub-gap regime. However, the conductance can be made to switch off as illustrated in Fig. 4(a) with $\lambda=0.8$, by tuning the out of-plane electric field to $\lambda>\mathrm{eV} / \Delta$. For $\mathrm{eV}>\Delta$ absence of Andreev reflection implies the peak value (for $\lambda=0$ ) to be at most $G / G_{0}=1$ [see Fig. 4(c)]. We note that the oscillatory dependence of $G / G_{0}$ on $\chi$ is in complete contradiction to the normal metal-insulator-superconductor junctions, where increasing the barrier strength always leads to the suppression of conductance ${ }^{20}$. 

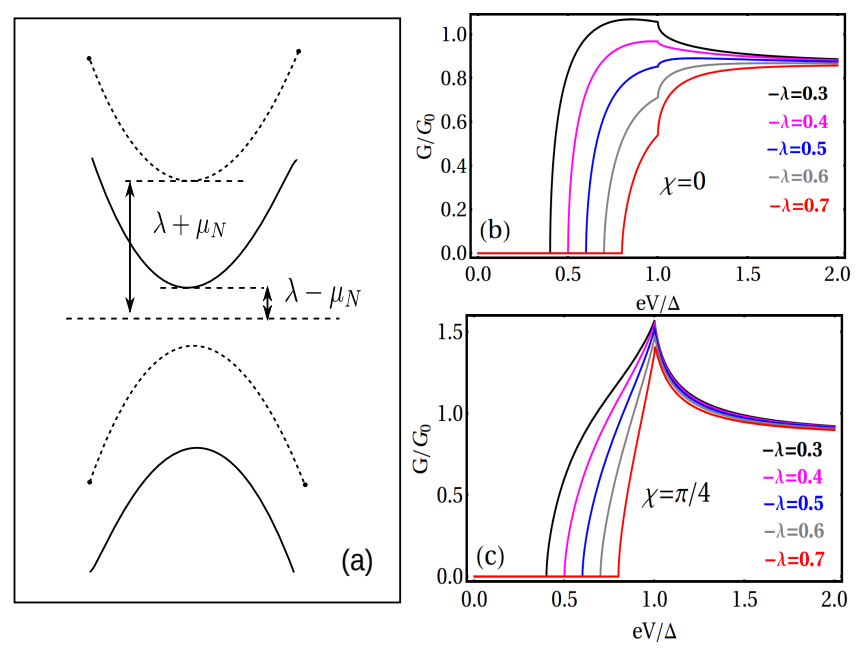

FIG. 5. (Color online)(a) BdG spectrum of normal silicene for $0<\mu_{N} / \Delta<\lambda<1$. Solid (dashed) lines represent particle (hole) like spectrum. (b)-(c) The tunnelling conductance acquires non-zero values for $e V>\lambda \Delta+\mu_{N}$.

For completeness we will consider a scenario wherein the chemical potential is non-zero, more specifically $0<$ $\mu_{N} / \Delta<\lambda<1$. Now, although electronic levels for $e V>\lambda \Delta-\mu_{N}$ exist, yet the conductance remains zero until the criterion $e V>\lambda \Delta+\mu_{N}$ is satisfied. This is due to the absence of Andreev reflection as there are no states available for hole reflection [see Fig. 5(a)]. We see this feature manifest itself in the $G / G_{0}$ vs $e V / \Delta$ plots in Figs. 5(b), (c). Note that unlike the $\mu_{N}=0$ case, now the transmission resonance criterion is not satisfied and the peak value of $G / G_{0}$ is smaller than 2 .
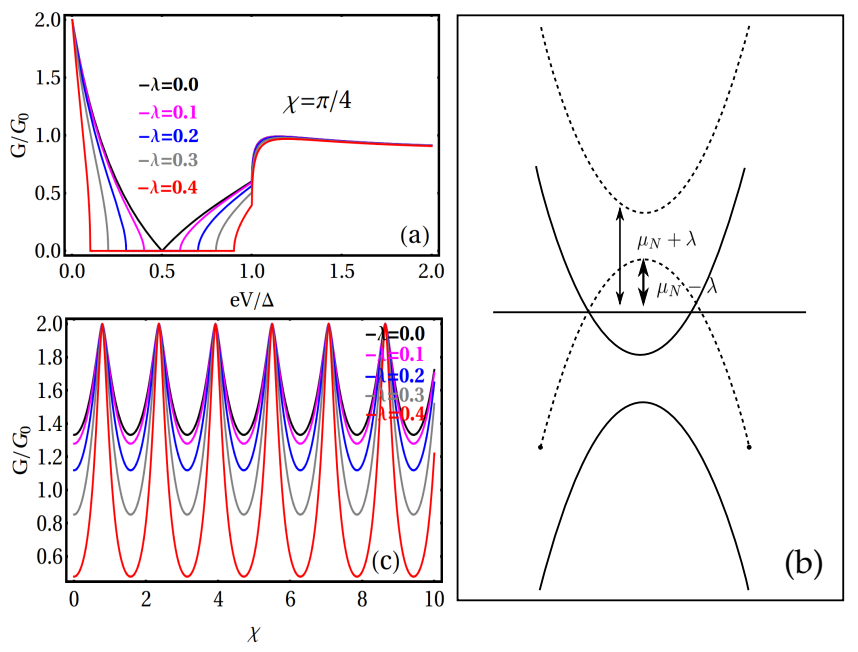

FIG. 6. (Color online)(a) In the moderately doped regime $\mu_{N}=0.5 \Delta, \mathrm{TC}$ vanishes between the ranges $\mu_{N}-\lambda \Delta<$ $e V<\lambda \Delta+\mu_{N}$. (b) BdG spectrum for $\lambda \Delta<\mu_{N}<l E_{Z}+\lambda_{S O}$. Andreev scattering is of retro type for $0<e V<\mu_{N}-\lambda \Delta$ and specular for $e V>\lambda \Delta+\mu_{N}$. (c) $G / G_{0}$ vs $\chi$ for $U_{0} \gg \Delta$ exhibits $\pi / 2$ oscillations.

\section{B. Moderately doped regime $\left(\mu_{N} \neq 0\right)$}

Here we will focus on moderate doping levels which we define as $\lambda \Delta<\mu_{N}<l E_{Z}+\lambda_{S O}$ and set $\mu_{N}=$ $0.5 \Delta$. It turns out that the profile of $G / G_{0}$ vs $e V$ plots as shown in Fig. 6(a) are markedly different from the earlier studied regimes. We find that the conductance at zero bias voltage starts out from a non-zero value and monotonically decreases to zero at $e V=\mu_{N}-\lambda \Delta$. The conductance remains zero till $e V=\lambda \Delta+\mu_{N}$, beyond which it increases monotonically till $e V=\Delta$.

For bias voltages in the range $0<e V<\mu_{N}-\lambda \Delta$, the Andreev scattering is accompanied by hole scattering of the usual retro type. On the other hand, when an electron with energy $\mu_{N}-\lambda \Delta<e V<\lambda \Delta+\mu_{N}$ is incident on the interface of NS junction it gets completely reflected back due to the absence of hole states [Fig. 6(b)]. In a further twist, an incident electron with energy in the range $e V>\lambda \Delta+\mu_{N}$ is again Andreev scattered due to the availability of hole states. However, the holes now undergo specular reflection due to the change in sign of the curvature of hole spectrum.

The oscillatory feature in the $G / G_{0}$ vs $\chi$ plots are shown in Fig. 6(c). For the simple case of $\lambda=0$ the expression for $r$ reduces to

$$
r=\frac{i\left(-1+e^{2 i \alpha_{e}}\right) \sin (2 \chi-\beta)}{2 \cos (2 \chi-\beta) \cos \left(\alpha_{e}\right)+2 i \sin (2 \chi-\beta)} .
$$

At zero bias, $\beta=\pi / 2$, the condition for transmission resonance reduces to $\chi=(n+1 / 2) \pi / 2$, so the first maxima is exhibited at $\chi=\pi / 4$.

\section{Highly doped regime}

In this subsection we present our tunneling conductance (TC) results for the highly doped regime i.e., $\mu_{N} \gg \Delta$. The mean field criterion, $\mu_{N}+U_{0} \gg \Delta$, is now automatically satisfied irrespective of the value of $U_{0}$. We will consider two scenarios, $U_{0} \gg \Delta$ and $U_{0} \ll \Delta$, and plot $G / G_{0}$ vs $e V / \Delta$ and vs $\chi$ in the two regimes. Since $\left(l E_{Z} \pm \lambda_{S O}\right) \sim \Delta$, both the bands in normal region are occupied and will contribute to the conductance.

Due to the large value of the chemical potential the $\mathrm{TC}$ is nearly insensitive to the variation in $\lambda$ and thus on the electric field applied perpendicular to the surface $E_{Z}$. However, the TC shows interesting behaviour in the two extreme regimes for $U_{0}$. For large $U_{0}$ the TC exhibits, as before, a change in the slope of $G / G_{0}$ vs $\mathrm{eV} / \Delta$ curve at $e V=\Delta$ for all barrier strengths [Fig. 7(a)]. Also, the $\pi / 2$ periodicity in the dependence of the TC on the barrier strength persists [Fig. $7(\mathrm{~b})]$. For small $U_{0}$ a similar change in slope at $e V=\Delta$ is present in $G / G_{0}$ vs $e V / \Delta$ plots, however, the TC now exhibits $\pi$ periodicity as a function of $\chi$ [see Figs. $7(\mathrm{c})-(\mathrm{d})]$.

When $U_{0}$ is large $\left(U_{0} \gg \Delta\right)$, there is a large Fermi wave-length mismatch between the normal and the superconducting side. In this scenario, we obtain the $\pi / 2$ 

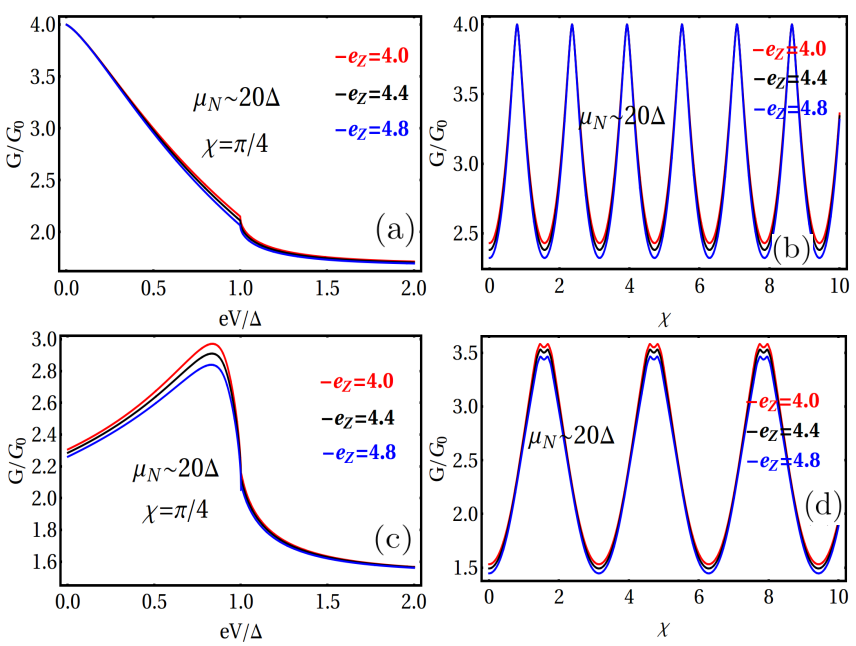

FIG. 7. (Color online) Plot of tunneling conductance as a function of bias voltage [(a) and (c)], and barrier strength $\chi$ [(b) and (d)] with $\lambda_{S O} / \Delta=4.0$ and $e_{Z}=l E_{Z} / \Delta$. Figures (a) and (b) correspond to $U_{0} \gg \Delta$, (c) and (d) correspond to $U_{0} \ll \Delta$.

periodicity in the dependence of the TC on the barrier strength $\chi$. On the other hand, for small $U_{0}\left(U_{0} \ll \Delta\right)$, Fermi wave-length mismatch turns out to be negligible between the two sides. This gives rise to the $\pi$ periodicity $^{21}$ in the behavior of TC with respect to $\chi$.

\section{SUMMARY AND CONCLUSIONS}

To summarize, in this article, we have presented a theory of tunneling conductance of a Normal-InsulatorSuperconductor (NIS) junction of silicene in the thin bar- rier limit. We have demonstrated that in this limit the tunneling conductance shows novel periodic behavior as a function of barrier strength. In particular, we note that the period of oscillation changes from $\pi / 2\left(U_{0} \gg \Delta\right)$ to $\pi\left(U_{0} \ll \Delta\right)$ with the variation of doping in the superconducting side of silicene. Moreover, for the undoped regime $\left(\mu_{N}=0\right)$, the external electric field $E_{z}$ can be used as a switch to tune the conductance from on to off condition. The latter is a unique feature of silicene.

As far as experimental realization of our silicene NIS set-up is concerned, it can be possible to realize a proximity induced superconducting gap in silicene by using $s$ wave superconductor like $\mathrm{Al}^{24}$. Typical spin-orbit energy in silicene is $\lambda_{\mathrm{SO}} \sim 4 \mathrm{meV}$ while the buckling parameter $l \approx 0.23 \AA^{5,10}$. Considering Ref. 24, typical induced superconducting gap in silicene would be $\sim 0.2 \mathrm{meV}$. For such induced gap, the change of periodicity of TC from $\pi / 2$ to $\pi$ may be possible to observe by changing the doping concentration from $\mu_{N} \sim 0.1 \mathrm{meV}$ to $\mu_{N} \sim 4 \mathrm{meV}$ for a barrier of thickness $\sim 10-15 \mathrm{~nm}$ and height $V_{0} \sim 500 \mathrm{meV}$ which can be considered as thin barrier. Also the typical range of the external electric field can be within $E_{z} \sim 180-200 \mathrm{~V} / \mu \mathrm{m}$ to use our set-up as a switch.

We expect our results to be qualitatively similar to the recently discovered two-dimensional materials like germenene, stanene ${ }^{4,25,26}$. Although the strength of Rashba spin-orbit coupling in these materials can be stronger than silicene $e^{5,10}$.
1 A. K. Geim and K. S. Novoselov, Nat. Materials 6, 183 (2007).

2 X. L. Qi and S. C. Zhang, Rev. Mod. Phys. 83, 1057 (2011).

3 M. Z. Hasan and C. L. Kane, Rev. Mod. Phys. 82, 3045 (2010).

4 M. Ezawa, J. Phys. Soc. Jpn. 84, 121003 (2015).

5 C. C. Liu, H. Jiang, and Y. Yao, Phys. Rev. B. 84, 195430 (2011).

6 B. Lalmi, H. Oughaddou, H. Enriquez, A. Kara, S. Vizzini, B. Ealet, and B. Aufray, Appl. Phys. Lett. 97, 223109 (2010).

7 P. D. Padova et al, Appl. Phys. Lett. 96, 261905 (2010).

${ }^{8}$ P. Vogt, P. D. Padova, C. Quaresima, J. Avila, E. Frantzeskakis, M. C. Asensio, A. Resta, B. Ealet, and G. L. Lay, Phys. Rev. Lett. 108, 155501 (2012).

9 N. D. Drummond, V. Zólyomi, and V. I. Falko, Phys. Rev. B. 85, 075423 (2012).

10 M. Ezawa, New J. Phys. 14, 033003 (2013).

11 L. Tao, E. Cinquanta, D. Chiappe, C. Grazianetti, M. Fanciulli, M. Dubey, A. Molle, and D. Akinwande, Nat. Nanotech. 10, 227 (2014).
12 M. Ezawa and N. Nagaosa, Phys. Rev. B. 88, 121401(R) (2013).

13 M. Ezawa, Eur. Phys. J. B 85, 363 (2012).

14 G. G. Guzmán-Verri and L. C. Lew Yan Voon, Phys. Rev. B. 76, 075131 (2007).

15 C. C. Liu, W. Feng, and Y. Yao, Phys. Rev. Lett. 107, 076802 (2011).

16 M. Ezawa, Phys. Rev. B. 87, 155415 (2013).

17 C. W. J. Beenakker, Rev. Mod. Phys. 80, 1337 (2008).

18 J. Linder and T. Yokoyama, Phys. Rev. B. 89, 020504(R) (2014).

19 C. W. J. Beenakker, Phys. Rev. Lett. 97, 067007 (2006).

${ }^{20}$ G. Blonder, M. Tinkham, and T. M. Klapwijk, Phy. Rev. B 25, 4515 (1982).

21 S. Bhattacharjee and K. Sengupta, Phys. Rev. Lett. 97, 217001 (2006).

22 S. Bhattacharjee, M. Maiti, and K. Sengupta, Phys. Rev. B 76, 184514 (2007).

23 A. S. Ruchi Saxena and S. Rao, Phy. Rev. B 92, 245412 (2015).

${ }^{24}$ H. B. Heersche, P. Jarillo-Herrero, J. B. Oostinga, L. M. K. 
Vandersypen, and A. F. Morpurgo, Nature 446, 56 (2007).

25 M. Dávila, L. Xian, S. Cahangirov, A. Rubio, and G. Le Lay, New J. Phys. 16, 095002 (2014).

${ }^{26}$ F.-f. Zhu, W.-j. Chen, Y. Xu, C.-l. Gao, D.-d. Guan, C.-h.
Liu, D. Qian, S.-C. Zhang, and J.-f. Jia, Nat. Mat. 14, 1020 (2015). 